\title{
Function and Localization of MicroRNAs in Mammalian Cells
}

\author{
A.K.L. LEUNG AND P.A. SHARP \\ Center for Cancer Research, Massachusetts Institute of Technology, Cambridge, Massachusetts 02139
}

\begin{abstract}
microRNAs (miRNAs) represent a large set of master regulators of gene expression. They constitute $1-4 \%$ of human genes and probably regulate $30 \%$ of protein-encoding genes. These small regulatory RNAs act at a posttranscriptional level—mediating translational repression and/or mRNA degradation - through their association with Argonaute protein and target mRNAs. In this paper, we discuss various mechanisms by which miRNAs regulate posttranscriptionally, including their subcellular localization. Recent results indicate that the majority of miRNA-targeted and thus translationally repressed mRNA is probably distributed in the diffuse cytoplasm, even though a small fraction is concentrated in subcellular compartments, such as processing bodies or stress granules; notably, the stress granule localization of Argonaute depends on the presence of miRNAs. Here we discuss the structural requirement of these subcellular compartments in light of their potential miRNA functions.
\end{abstract}

The miRNAs are a class of noncoding RNAs found in animals and plants, whose mature products are approximately 21-22 nucleotides in length (Bartel 2004; Kim 2005). These RNAs are predicted to regulate about $30 \%$ of mammalian mRNAs through interactions with their 3'UTRs (untranslated regions) (Lewis et al. 2005; Rajewsky 2006). The binding of miRNAs can result in translational repression and/or degradation of mRNAs (Filipowicz et al. 2005; Valencia-Sanchez et al. 2006). We discuss here the various functions of miRNAs and their possible cytoplasmic locale in mammalian cells.

\section{MIRNA-MEDIATED TRANSLATIONAL REPRESSION}

The role of miRNA was first demonstrated as the translational repressor by its founding member lin-4 in the nematode Caenorhabditis elegans (Lee et al. 1993; Wightman et al. 1993). Genetic analysis showed that the gene product of lin- 4 acted as a negative regulator of LIN14 expression after larval stage L1, and the 3'UTR of the lin-14 mRNA was required for this regulation (Arasu et al. 1991). After the discovery of the gene product of lin-4 as a 22-nucleotide RNA and the potential binding sites at the 3'UTR of lin-14 mRNA, the miRNA:mRNA base-pairing was realized to be crucial in regulating lin-14 expression (Lee et al. 1993; Wightman et al. 1993). The 3'UTR of lin14 mRNAs contains seven lin-4 miRNA-binding sites, which are conserved in Caenorhabditis briggsae. lin-14 mRNAs with mutations in all of these binding sites can no longer be down-regulated by lin-4 (Ha et al. 1996). It was initially found that the steady-state level of lin-14 transcript was not reduced more than twofold by the lin-4 (although a recent report detected a fivefold reduction; Bagga et al. 2005; and see below), whereas its protein level was reduced by approximately tenfold from larval stage L1 to L2 (Olsen and Ambros 1999). Furthermore, neither the transcription rate nor the status of polyadenylation for lin-14 mRNA changed from L1 to L2 (Olsen and Ambros 1999). This evidence points to the role of lin-4 as a translational repressor of lin-14 mRNA.
The mechanisms by which lin-4 suppresses the expression of lin-14 mRNA were investigated. Biochemical analysis suggested that both lin-4 miRNA and lin-14 mRNA cosedimented with polyribosomes (Olsen and Ambros 1999). A similar polyribosomal association of miRNA is also observed in mammalian cells (Kim et al. 2004; Nelson et al. 2004; Petersen et al. 2006). However, another substantial fraction of miRNAs do not cosediment with the polyribosomes, but with a fraction slower than monosomes (Nelson et al. 2004). This more slowly sedimenting fraction is typically devoid of target mRNA, probably representing miRNA-containing ribonucleoparticles (miRNPs) in excess to target mRNAs or those miRNAs that dissociated from their target mRNAs during isolation. Evidence showing that the polyribosome-associated miRNA target mRNAs are engaged in some form of active translation has been reported, for example, human K-ras and lin-28 mRNAs that are targeted by let-7 miRNA (Nelson et al. 2004; Maroney et al., this volume) or a model luciferase mRNA targeted by a partially complementary small interfering RNA (siRNA) (Petersen et al. 2006). In both cases, short pulses of the peptidyl transferase inhibitor puromycin released the repressed mRNAs from the polyribosomes. Conversely, miRNA-mediated translational repression was proposed to act at the stage of initiation. When targeted by let-7 miRNA or directly tethered with Ago2, a model luciferase mRNA in the polysome profile was significantly shifted toward the monosome fraction from the polyribosomes (Pillai et al. 2005). Thus, miRNA-mediated translational repression can probably occur both at a step postinitiation or at initiation (Fig. 1).

\section{Repression Postinitiation}

On the basis of the following observations, our laboratory proposed that miRNA might act to promote abortive termination, resulting in "ribosome drop-off" during elongation of translation (Petersen et al. 2006): First, the readthrough rate of a termination codon artificially introduced between two open reading frames is less efficient in the presence of a miRNA that targets their shared 


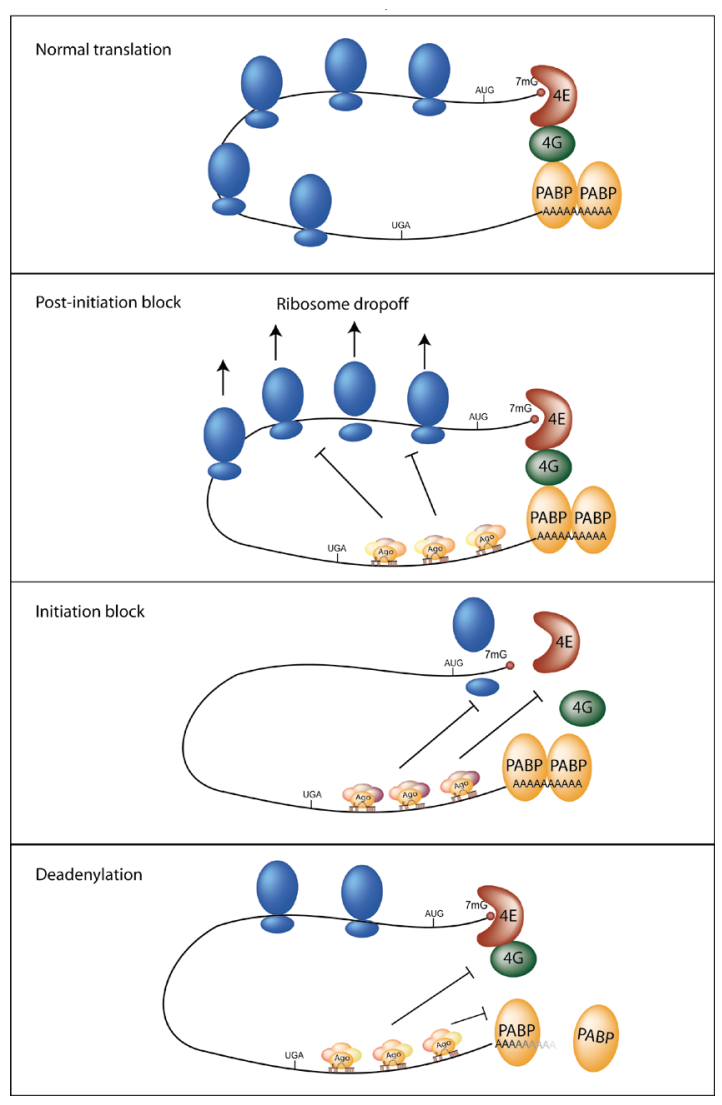

Figure 1. Different modes of miRNA-mediated translational repression.

3'UTR, suggesting that more termination events occur in the presence of miRNA. Second, miRNA-targeted mRNAs dissociate from ribosomes more readily than nontargeted control mRNA when translation initiation is inhibited, suggesting an enhanced rate of termination or release of ribosomes. Third, miRNA can still repress the translation of mRNAs that bypass any requirement for a typical initiation, including cap binding, scanning, and binding of multiple initiation factors; this involved the use of an internal ribosome entry site (IRES) from a cricket paralysis virus intergenic region (CrPV-IGR). These data, together with the puromycin sensitivity of the polyribosome profile of the target mRNA, suggest that miRNA acts at a step postinitiation. In particular, we propose that it might modulate the processivity of translation, that is, an enhancement of the abortive termination rate. This abortive translational mechanism ostensibly wastes energy in a nonproductive manner; however, when relieved, these types of inhibitions with continual translation could result in rapid modulation of the translation rate, and hence increase in the protein yield within a short period of time.

Postinitiation repression is not restricted to miRNAtype translational repression. For proper embryonic development, $4 \%$ of Nanos mRNAs are exclusively localized to the posterior pole of the Drosophila embryo, whereas the remaining $96 \%$ of unlocalized mRNAs must be translationally repressed elsewhere (Gavis and
Lehmann 1994); the repression is mediated by an element at the 3'UTR. These unlocalized Nanos mRNAs are associated with polyribosomes in a puromycin-sensitive manner (Clark et al. 2000). Interestingly, the Nanos mRNA-associated polyribosomes are capable of completing the elongation phase of the translation cycle in in vitro extracts made from a specific embryo strain where the mRNAs are all unlocalized and translationally repressed. This suggests a regulatory mechanism that does not involve a stable arrest of the translation cycle, similar to what we observed in miRNA-mediated translational repression.

\section{Repression at Initiation}

Most translational initiation begins with the recognition of the mRNA cap, where initiation factors are assembled together with Met-tRNA and a 40S ribosome to form a preinitiation complex (Pestova et al. 2001; Sonenberg and Dever 2003). This preinitiation complex then scans across the message to the initiation codon, resulting in the joining of other initiation factors and finally the $60 \mathrm{~S}$ ribosome. A luciferase reporter with three artificial bulged let-7-binding sites, when transfected into HeLa cells in which let-7 miRNA is abundant, results in approximately $30 \%$ reduction at the mRNA level and tenfold reduction in the protein level (Pillai et al. 2005). Polysome profiling of the reporter mRNA showed that a major fraction shifts toward the top of the gradient as compared to a control where the anti-let$72^{\prime} \mathrm{O}-\mathrm{Me}$ oligonucleotide was cotransfected into the cells. This suggests that the let-7 miRNA is responsible for the shift in the polysome profile of the target mRNA. This shift in polysome profile with miRNA-mediated repression is similarly observed when Ago2 is tethered to the 3'UTR of the luciferase reporter (Pillai et al. 2005). In both cases, the shift in the profile results in an increase of $25 \%$ of reporter mRNA to the top of the gradient; however, there are still about $50 \%$ of the signals notably remaining in the polyribosome fraction in the experiment. The moderate shift in profile and the remaining fraction in the polyribosomes suggest a decrease in efficiency, rather than a total block, at translation initiation.

Other experiments using a target mRNA with defined initiation requirements have been used to address which steps of translation are repressed by miRNAs (Humphreys et al. 2005; Pillai et al. 2005). Pillai et al. (2005) reported that miRNA repression did not occur when translation was driven by tethering initiation factors, such as the cap-binding protein eIF4E or its binding partner eIF4G, upstream of an internal open reading frame, suggesting that miRNA might act at initiation, most likely upstream of the step where eIF4E recruits eIF4G. However, in these cases, it is not known whether artificially tethering multiple copies of initiation factor will outcompete the repression machinery or if the rate of artificial initiation is so strong that it masks an otherwise subtle effect such as a decrease in processivity. Another way to modulate the initiation dependence on cap is to use different viral IRES sequences, which differ by their initiation factor requirement (Humphreys et al. 2005; Pillai et al. 2005). When transfecting in-vitro-synthesized RNA 
with CrPV or hepatitis $\mathrm{C}$ virus (HCV) IRES, miRNAs no longer repress their translation. However, miRNA-mediated translational repression still occurs when DNA constructs encoding messages with the CrPV or HCV IRES were transfected into cells (Petersen et al. 2006), potentially suggesting that the "nuclear history" of an mRNA might be important for miRNA-mediated repression and/or that the protein associated with the in-vivo-synthesized mRNA may be different from those transfected after in vitro synthesis. In either case, if miRNA-mediated repression acts at the initiation step, it is yet to be reconciled with the fact that the bulk of target-associated miRNA cosediments with polyribosomes (Kim et al. 2004; Nelson et al. 2004).

Both the cap and poly(A) tail seem to be required, but are not sufficient by themselves, for the full repression mediated by miRNAs (Humphreys et al. 2005; Pillai et al. 2005). Factors binding at the cap and poly(A) tail are known to synergistically interact with each other to enhance translation. Such interactions are commonly modulated by elements at the 3'UTR that result in the lowering of the initiation efficiency (Gebauer and Hentze 2004). If miRNAs can alter the polyadenylation status of the message (see below), the disruption of the interaction between the cap and poly(A) tail might provide yet another mechanism for miRNA-mediated translational repression (Fig. 1, bottom panel).

\section{MIRNA-INDUCED DEGRADATION OF MRNA}

Numerous examples demonstrate that miRNAs repress translation with little or no change of steady-state mRNA levels (Brennecke et al. 2003; Saxena et al. 2003; Zeng et al. 2003; Chen et al. 2004; Doench and Sharp 2004; Poy et al. 2004; Cimmino et al. 2005; Pillai et al. 2005). Yet, a growing number of examples show that miRNAs also cause a reduction in mRNA levels (Yekta et al. 2004; Bagga et al. 2005; Davis et al. 2005; Jing et al. 2005; Wu and Belasco 2005; Behm-Ansmant et al. 2006; Giraldez et al. 2006; Gupta et al. 2006; Rehwinkel et al. 2006; Wu et al. 2006). In most cases, the reduction in RNA level is approximately 1.5 - to 2 -fold as measured by microarrays after transfection with miRNAs in the form of an siRNA duplex (Lim et al. 2005). Consistent with these transfection results, recent bioinformatic comparisons of miRNA expression and predicted target mRNA levels show an inverse relationship (Farh et al. 2005). Currently, there are three reported types of miRNA-induced degradations (Fig. 2 ): The first requires extensive complementarity throughout the miRNA, the second requires complementarity particularly at the seed region (i.e., 2nd-8th nucleotides) of miRNA, and the third is based on the complementarity at a central AU-rich region of miRNA (Fig. 2).

\section{Endonucleolytic Cleavage}

Unlike the situation in plants, mammalian miRNAs rarely have extensive complementarity with their targets. So far, there are two reported cases: The first case involves miR-196, where the whole 22-nucleotide sequence has conserved complementarity to a region in the 3 'UTR of the HoxB 8 mRNA; the complementarity

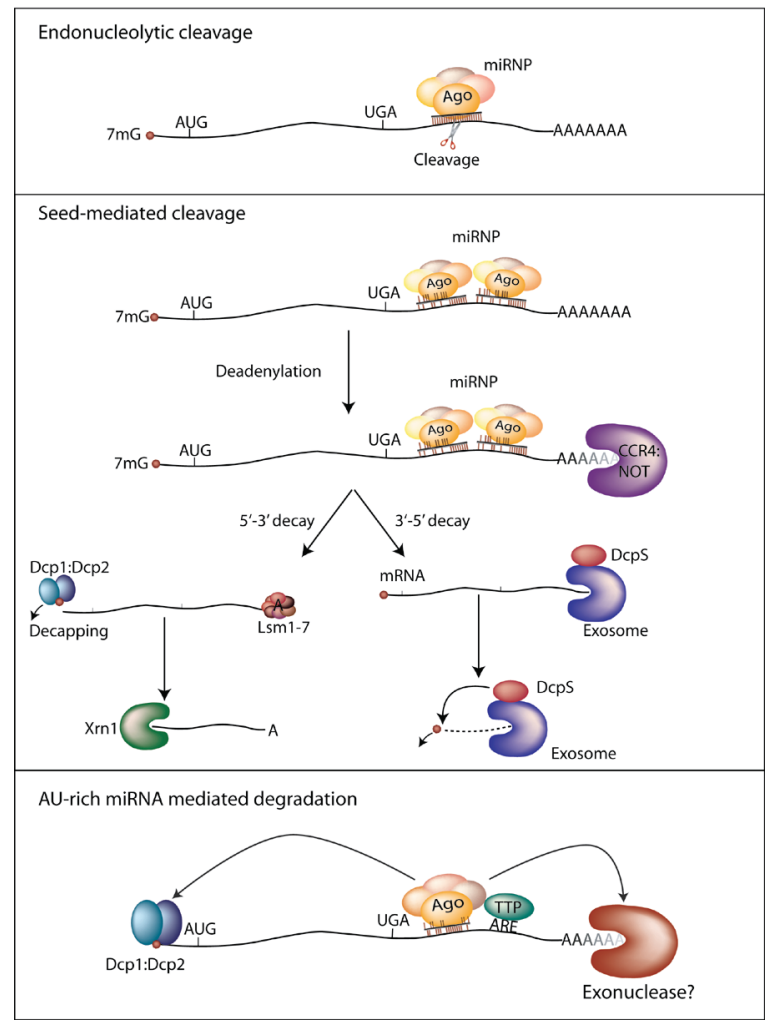

Figure 2. Different modes of miRNA-mediated mRNA degradation.

includes a non-Watson-Crick base-pair G:U wobble at the fifth position (Yekta et al. 2004; Hornstein et al. 2005). The other case involves several miRNAs encoded in the Dlk1-Gtl2 imprinted domain region. These miRNAs are processed from a transcript that is antisense to a paternally expressed Rtll/Peg11 mRNA; therefore, the antisense transcript, by definition, possesses multiple perfectly complementary binding sites for these miRNAs. The presence of these miRNAs results in the cleavage of their targets between the corresponding 10th and 11th positions of the miRNA, reminiscent of an RNA interference (RNAi)-like cleavage event supposedly by Argonaute 2 (Ago2). Currently, Ago2 is the only known Argonaute family member in mammalian cells that possesses endonucleolytic (or "slicer") capability (Liu et al. 2004). However, when complexed with other Argonaute family members, these miRNAs are not thought to mediate direct cleavage, but their associations with particular targets should mediate translational repression and perhaps degradation of the transcripts.

\section{"Seed"-mediated Degradation}

miRNA and siRNA silencing pathways have been shown to have some common activities. For example, endogenous miRNAs can direct cleavage of exogenously expressed targets that contain sites of extensive complementarity, and siRNAs can function as miRNAs in mediating translational repression (Hutvagner and Zamore 2002; Doench et al. 2003; Saxena et al. 2003; Zeng et al. 
2003). Another aspect of siRNA behaving as an miRNA pathway was revealed when it was observed that there is 1.5- to 2-fold degradation of multiple mRNAs (the socalled "off" target) that does not have a perfect complementary binding site with the siRNA (Jackson et al. 2003). The major determinant for this type of degradation turns out to be the propensity of the $5^{\prime}$ end of the siRNA pairing with these "off-targets" (Lin et al. 2005; Birmingham et al. 2006; Jackson et al. 2006a). This is strikingly similar to the seed region requirement for miRNA-mediated mRNA degradation (Lim et al. 2005). This mode of degradation does not involve RNAi-like endonucleolytic cleavage activity because the sequences of mRNA degradation products are not ended at the corresponding 11th position of miRNA (Bagga et al. 2005; Wu et al. 2006).

The underlying mechanism for this type of degradation is still not clear, but we can draw parallels from the study of the "off-target" effect: (1) This effect is observed at a low level of transfected siRNA and is probably the result of a direct interaction between siRNA and target mRNA, as it is observed shortly after transfection (Jackson et al. 2003, 2006a). The degree of off-target degradation can be partially inhibited by adding a 2'O-methyl ribosyl substitution at position 2, suggesting that this substituted position might be crucial in some steps leading to mRNA degradation (Fedorov et al. 2006; Jackson et al. 2006b). (2) This effect is not observed in cells depleted of either nonslicer Agol or slicer Ago2, suggesting that endonucleolytic capability of Argonaute protein might not be a prerequisite (Fedorov et al. 2006). (3) The degree of offtarget degradation is greater with multiple seed sites and is also dependent on sequence context surrounding the complementary site (Lin et al. 2005). (4) This effect is observed more often if the complementary site is in the 3'UTR, rather than the coding sequence or 5'UTR (Birmingham et al. 2006; Jackson et al. 2006a).

Other lines of evidence concerning miRNA-induced degradation come from genetic studies in Drosophila and zebra fish (Behm-Ansmant et al. 2006; Giraldez et al. 2006; Rehwinkel et al. 2006). When depleted of the primary component, such as dAgol or GW182, of the miRNA pathway in Drosophila S2 cells, the RNA level of predicted miRNA targets increases as measured by microarrays (Behm-Ansmant et al. 2006); therefore, these targets are most likely degraded by miRNA in addition to being translationally repressed. When these predicted miRNA targets are fused to the 3'UTR of luciferase, these targets are rapidly degraded with cotransfection of the corresponding miRNA due to an increase of the deadenylation rate. Subsequent studies have shown that this type of mRNA degradation requires both CCR4:NOT deadenylase and DCP1:DCP2 decapping complexes (BehmAnsmant et al. 2006). Tethering of GW182 to a luciferase mRNA alone can bypass the requirement of dAgol in promoting decay, suggesting that the binding of GW182 to the message through the dAgol/miRNA complex marks the transcript as a target for degradation (BehmAnsmant et al. 2006). In many systems, deadenylation is often the first step toward degradation of the transcript, which is followed by two pathways (Fig. 2) - one involving decapping followed by $5^{\prime} \rightarrow 3^{\prime}$ degradation by an exoribonuclease such as Xrn1, and the other beginning with $3^{\prime} \rightarrow 5^{\prime}$ decay by the exosome, followed with cap removal by the scavenger DcpS (Parker and Song 2004; Fillman and Lykke-Andersen 2005). The idea of accelerated deadenylation by miRNA is reinforced in the study of zebra fish (Giraldez et al. 2006). miR-430, a highly expressed miRNA in early zebra fish embryogenesis, increases the deadenylation rate of its targets at the onset of zygotic gene expression. Given that most of the miR430 targets are maternal mRNAs, the resultant approximately fivefold reduction of these targets underlies the clearance of maternal mRNAs. Interestingly, miRNAinduced deadenylation still occurs in the absence of translation, suggesting that deadenylation is not necessarily a secondary effect of nonproductive translation (Giraldez et al. 2006). It should, however, be stressed that in both cases, deadenylation/degradation was only studied for those miRNA targets that displayed measurable changes in mRNA level, and the phenomenon might not hold true for all miRNA targets.

Wu et al. (2006) also described similar miRNAinduced deadenylation in mammalian cells in a study of miR $125 \mathrm{~b}$ and its target lin-28. These authors reported that the down-regulation of lin-28 expression is due to both translational repression and degradation by accelerated deadenylation. The degradation requires that the miRNAbinding site be located prior to the polyadenylation signal on the message (Wu and Belasco 2005). As observed in zebra fish, the miRNA-induced deadenylation of the target occurs even in the presence of a large stem-loop in the 5 'UTR that abolished $99 \%$ of protein synthesis. Conversely, translational repression still occurs in mRNA containing a histone stem-loop that replaces the poly(A) tail, suggesting that translational repression and deadenylation induced by this process are not mutually dependent.

\section{"AU-rich miRNA"-mediated Decay}

A genome-wide RNAi screen revealed that AU-rich element (ARE)-mediated decay requires Dicer in Drosophila $\mathrm{S} 2$ cells, and this requirement was also demonstrated in human HeLa cells (Jing et al. 2005). This ARE-mediated decay involves $m i R-16$, which has previously been shown to mediate translational repression (Cimmino et al. 2005). However, this miRNA contains an UAAAUAUU sequence between the 10th and the 17th position, which is complementary to a typical ARE. The mRNA decay requires both the ARE-binding protein tristetraprolin (TTP) and miR-16. However, TTP does not directly bind miR-16, but instead interacts through protein-protein interaction with human Ago2/Ago4 that binds the miRNA. It is noteworthy that the binding site in the target mRNA is complementary to the central region of the miRNA, rather than the $5^{\prime}$ region, as observed in the seedmediated cleavage. The significance of this differential binding mode remains to be determined.

\section{WHERE ARE THESE FUNCTIONS LOCALIZED?}

Where are these various functions of miRNAs localized in the cells? Are they related to subcellular compartments in the cytoplasm? Interestingly, miRNAs, 
miRNA-targeted mRNAs, and proteins associated with their activities have been found to localize to specific subcellular structures. We first examine the evidence of the localization of miRNAs, miRNA targets, and their associated proteins and discuss the significance of colocalization of these factors in specific structures.

\section{Localization of miRNAs}

In animals, the primary transcript of miRNA (primiRNA) is transcribed by RNA polymerase II and sequentially processed to an approximately 70-nucleotide pre-miRNA intermediate in the nucleus by class III ribonuclease Drosha (Kim 2005). The pre-miRNA intermediate can be folded in silico into a stem-loop hairpin with a short ( $\sim 2$ nucleotides) 3 ' overhang. The resulting "minihelix" structure of the pre-miRNA intermediate is recognized by Exportin 5 and exported to the cytoplasm in a RanGTP-dependent manner. The double-stranded pre-miRNA intermediate is then processed by another class III ribonuclease enzyme Dicer in the cytoplasm, where it generates the mature single-stranded miRNA $(\mathrm{miR})$ and the opposite strand $\left(\mathrm{miR}^{*}\right)$. The opposite strand presumably undergoes rapid degradation, supported by its usual absence in small RNA cloning or northern blot analysis. Subcellular fractionation studies have confirmed that the active single-stranded form of miRNA is primarily localized in the cytoplasm (Fig. 3).

Several methods have been used to identify the specific location of mature miRNAs in the cytoplasm (Jakymiw et al. 2005; Pillai et al. 2005; Bhattacharyya et al. 2006). Pillai et al. (2005) microinjected an in-vitro-transcribed, fluorescently labeled artificial precursor of let-7a into nuclei and found that $21.8 \%$ of the cytoplasmic fluorescent signals localized at $(13.7 \%)$ or near $(8.1 \%)$ process-

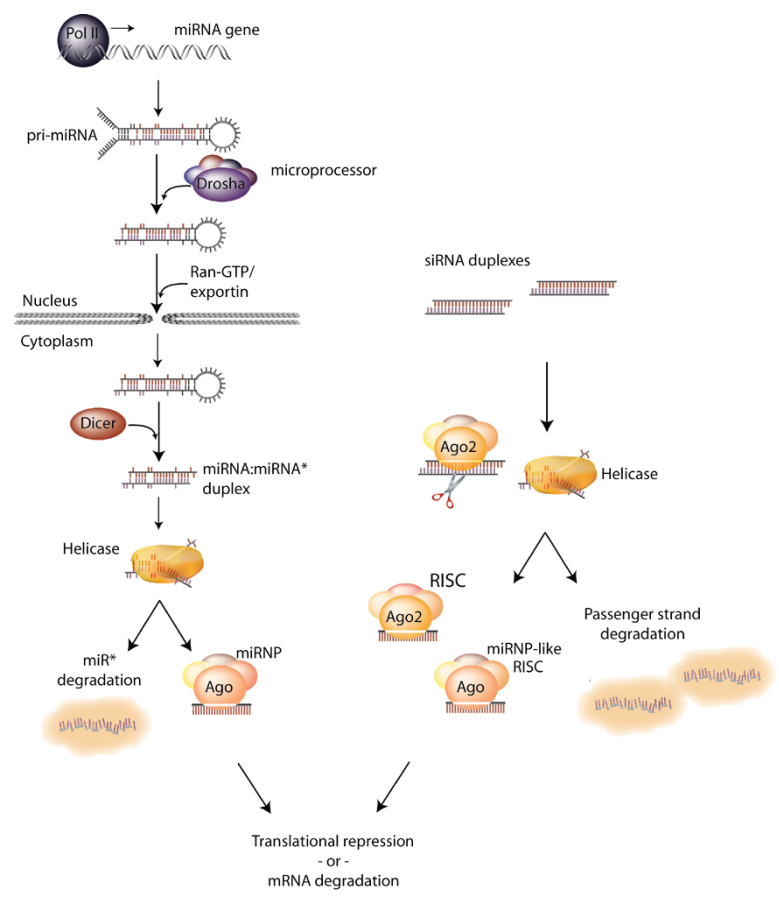

Figure 3. miRNA and siRNA pathways lead to translational repression or mRNA degradation. ing bodies (PBs), a known site for mRNA decay. However, in this approach, both the active miR strand and the $\mathrm{miR}^{*}$ strand of miRNA were equally labeled and therefore cannot easily be distinguished from each other. The signal observed at PBs could represent the degradation site for $\mathrm{miR}^{*}$, rather than the active strand. Furthermore, it is not possible to determine the fraction of the labeled intracellular RNA that is processed to a mature miRNA. Jakaymiw et al. (2005) labeled the antisense strand of an siRNA duplex and found that the labeled strand accumulated in PBs. In this case, it is similarly difficult to be certain that the labeled antisense strand is the one loaded into the RNA-induced silencing complex (RISC) as contrasted to the one targeted for decay. Whether PB is the site for decay of the miR* strand or the passenger strand remains unclear. In situ hybridization using locked nucleic acid (LNA) probes against a highly abundant miRNA, $m i R 122$, followed by signal amplification, also revealed the accumulation of the signals at PBs in hepatocytes (Bhattacharyya et al. 2006). Thus, several lines of evidence suggest that miRNAs might associate with PBs; the extent of this association is not clear. Furthermore, since PBs are free of ribosomal subunits (Anderson and Kedersha 2006), it is difficult to reconcile this subcellular location with several observations showing that a substantial fraction of miRNA associates with polyribosomes (Kim et al. 2004; Nelson et al. 2004).

To study miRNA localization, we aimed to avoid the ambiguous detection of inactive $\mathrm{miR} / \mathrm{miR} *$ duplexes or the $\mathrm{miR}^{*}$ strand and to quantitate the signal in the linear range. To this end, we have modified a well-characterized siRNA duplex that has been shown to function as a miRNA. The antisense strand is labeled with tetramethylrhodamine (TAMRA) at its $3^{\prime}$ end, and the $5^{\prime}$ phosphate group of the sense strand is substituted with black hole quencher 2 (BHQ2). In this way, the fluorescence is quenched in the siRNA duplex form, thereby enhancing the signal detection from the active single strand. Since the $5^{\prime}$ phosphate group is a prerequisite for RISC loading (Tomari et al. 2004; Rivas et al. 2005), the substitution of the phosphate group with the quencher presumably favors the selection of the labeled antisense strand for loading into the RISC complex. Using this modified siRNA, we did not observe any signal at PBs that was higher than the cytoplasmic background; instead, the signal was diffusively distributed throughout the cytoplasm (Leung et al. 2006).

\section{Localization of Target mRNA}

To localize repressed targets, Liu et al. (2005a) used green fluorescent protein (GFP)-tagged MS2-binding proteins to track luciferase mRNA that has 24 copies of MS2binding sites behind either two tandemly arranged let-7-binding sites or six CXCR4-binding sites. The target was localized to PBs in a miRNA-dependent manner. Furthermore, Pillai et al. (2005) quantitated the in situ hybridization signal of let-7-targeted luciferase mRNA and, similar to let-7, these authors found that $20.7 \%$ localized at (13.0\%) or near (7.7\%) PBs. We also found similar localization of the mRNA targets at or near PBs. Diffuse signal was also measured throughout the cytoplasm using the in situ hybridization method (Leung et al. 2006). 


\section{Localization of Protein Components}

Argonaute proteins bind to the miRNA forming the core component of RISC or miRNP for translational repression, endonucleolytic cleavage, and other types of mRNA degradation (Carmell et al. 2002). In human cells, there are four different Argonaute family members (Ago1-4), all of which accumulate at PBs (Jakymiw et al. 2005; Liu et al. 2005a,b; Pillai et al. 2005; Sen and Blau 2005; Chu and Rana 2006; Leung et al. 2006). Together with the colocalization of miRNA and its target, these data suggest a potential role of the $\mathrm{PB}$ as a site for miRNA function. This potentially new role of the $\mathrm{PB}$, apart from being the site for mRNA decay, was reinforced by the fact that depletion of another PB component GW182, which physically associates with Argonaute through a protein-protein interaction, impaired PB formation and miRNA-mediated translational repression activity (Liu et al. 2005a).

To assess the extent of localization of Argonaute protein to PBs, we established a HeLa cell line that stably expresses GFP-tagged human Argonaute 2 (Leung et al. 2006). Consistent with the published data, we found that the enhanced GFP (EGFP)-Ago2 signal intensity is on average tenfold higher at PBs than the neighboring cytoplasm. By quantitation, however, we found that approximately only $1 \%$ of the total cytoplasmic signal of Argonaute is localized to PBs, whereas the majority is diffuse throughout the cytoplasm. Moreover, using photoactivation and photobleaching techniques (Lippincott-Schwartz et al. 2003), we found that the off-rate of EGFP-Ago 2 at PBs is very slow. Similar observations of a slow rate of exchange was observed at PBs for both Argonaute-associated protein GW182 and the cap-binding protein eIF4E (Andrei et al. 2005; Kedersha et al. 2005). This is in contrast to the fast exchange rate observed for other PB components such as decapping enzymes Dcp1a/2 and decapping activator component Lsm6, suggesting that PBs comprise heterogeneous mRNPs with differential rates of exchange (Andrei et al. 2005; Kedersha et al. 2005; Leung et al. 2006). These quantitative analyses suggest that only small amounts of Argonaute proteins localize to $\mathrm{PBs}$, and they rarely exchange with the cytoplasm once localized there. So, apart from PBs, could miRNA function elsewhere?

\section{CYTOPLASMIC STRUCTURES FOR MIRNA FUNCTIONS?}

At least two cytoplasmic structures have been reported to associate with repressed mRNAs - processing bodies (PBs) and stress granules (SGs) (Anderson and Kedersha 2006).

\section{Processing Bodies}

PBs are well characterized in yeast, but much less studied in mammalian cells. Yeast and mammalian PBs share several characteristics: (1) They contain components that are involved in mRNA decapping and $5^{\prime} \rightarrow 3^{\prime}$ exonucleolytic decay, but lack ribosomal subunits (Sheth and Parker 2003; Cougot et al. 2004; Andrei et al. 2005; Anderson and Kedersha 2006), (2) their integrities depend on the presence of nontranslating mRNAs (Cougot et al. 2004; Teixeira et al. 2005), and (3) their structures dissociate in the presence of drugs that stabilize the association of mRNA with ribosomes, such as emetine and cycloheximide (Sheth and Parker 2003; Cougot et al. 2004; Kedersha et al. 2005; Teixeira et al. 2005). On the other hand, yeast and mammalian PBs differ in terms of composition, and in their response to growth and stress conditions. For example, only mammalian PBs contain the deadenylase CCR4 (Sheth and Parker 2003). Mammalian PBs increase in size and number when cells are proliferating or during the $\mathrm{S} / \mathrm{G}_{2}$ phase of the cell cycle, whereas yeast PBs increase when starved or grown at high density (Yang et al. 2004; Teixeira et al. 2005). With stress, yeast PBs, but not mammalian PBs, increase in size.

\section{Stress Granules}

In mammalian cells, under stress conditions, newly assembled structures known as stress granules (SGs) are formed where translationally stalled messages accumulate (Kedersha et al. 2005; Teixeira et al. 2005). Translationally repressed mRNAs localized in yeast PBs can leave and reenter the polyribosome pool (Brengues et al. 2005), but a similar phenomenon has not been demonstrated for mammalian PBs. Instead, SGs are thought to function as triage sites where mRNAs are sorted for future degradation, storage, or translation (Kedersha and Anderson 2002). SGs are known to be sites where translationally stalled poly $(\mathrm{A})^{+}$mRNAs accumulate when cells experience stress, such as heat shock, osmotic stress, or oxidative stress, or when translation initiation is specifically inhibited. PBs and SGs are known to be dynamically associated with, and often juxtaposing, each other. Although they share components, such as the cap-binding protein eIF4E and the translational repressor rck/p54, not all PB components are found in SGs, or vice versa (Anderson and Kedersha 2006). The close interaction observed between SGs and PBs in mammalian cells suggests that transcripts may be exported from SGs to PBs for degradation (Kedersha et al. 2005). Whether miRNAs, target mRNAs, and Argonaute proteins localize to SGs has not been determined. We report here the first evidence for the localization of miRNA and its target to SGs and that Argonaute protein localization to SGs is miRNAdependent (Leung et al. 2006).

\section{miRNA, mRNA Target, and Argonaute also Localize to Stress Granules}

We found that upon limiting translation initiation with an eIF4A-specific inhibitor, Hippuristanol, EGFP-Ago2 remained localized to $\mathrm{PBs}$ at the same level, but approximately $2-3 \%$ of the total fluorescence signal now localized to SGs. Quantitatively, there was about threefold more EGFP localized to SGs than to $\mathrm{PBs}$ after Hippuristanol treatment. Given that PB localization did not change with treatment, additional Argonaute proteins were redistributed from the diffuse cytoplasm to these distinct structures. In contrast to the relative immobility of 
Argonaute proteins demonstrated at PBs, Argonaute proteins at SGs were more dynamic as shown by photobleaching experiments. The fluorescence at a single photobleached spot at an SG can be fully recovered to its initial intensity level within 6 minutes, whereas the photobleached spot at a PB never recovered during this period. Furthermore, these preformed, Argonaute-positive SGs dissociate in the presence of Emetine, a drug that stabilizes the association of mRNA and polyribosomes, suggesting a dynamic relationship between SGs and polyribosomes. These treatments do not dissociate EGFP-Ago2 from PBs.

Upon SG induction, we detected a 1.5-fold enrichment of miRNAs at SGs as compared with the neighboring cytoplasmic signal, but no enrichment was observed at PBs, using our quencher-TAMRA-based detection method. Interestingly, miRNA targets also accumulated in SGs. These observations prompted us to postulate that Argonaute protein localization to SG is dependent on miRNA. To test this hypothesis, we transfected EGFPAgo 2 into three clonal mouse embryonic stem (ES) cell lines that contained a mutant nonfunctional form of Dicer and hence lacked mature miRNAs. We found that EGFPAgo2, although still observed in PBs, no longer associated with SGs. However, in the presence of exogenously supplied let-7a miRNA transfected in the form of siRNA, EGFP-Ago2 association with SGs was restored. This strongly suggests that the Argonaute localization to SGs is miRNA-dependent.

\section{Do We Need Any Structure for miRNA Function?}

Given that microscopically visible SGs are not observed in cells when translation initiation is not limiting, these macrostructures themselves are most likely not required for miRNA functions. However, upon stress, the accumulation of miRNA and its target at SGs and the miRNA-dependent localization of Argonaute proteins to SGs might reflect a potential relationship between miRNAs and SGs. SGs could possibly represent the aggregation of submicroscopic miRNPs that were formerly involved in translational repression of the target mRNA. Interestingly, multiple SG components FMRP, TTP, and HuR are known to interact with the Argonaute protein (Caudy et al. 2002; Jing et al. 2005; Bhattacharyya et al. 2006). Therefore, another possibility is that these newly assembled SGs preferentially recruit particular miRNA targets through protein-protein interaction for specific functions during stress.

Several lines of evidence indicate that microscopically visible PBs are also not required for translational repression, endonucleolytic activity, and ARE-mediated mRNA decay. For example, depletion of the PB component Lsm1 impairs PB formation but does not affect miRNA-mediated translational repression activity or endonucleolytic activity (Chu and Rana 2006). Therefore, the miRNA and siRNA pathways appear to be intact in these unstressed cells, where no microscopically visible PBs and SGs are observed. Similarly, no negative effects on ARE-mediated decay or deadenylation were observed in the absence of visible PBs when p54/rck or Dcp2 was overexpressed (Fenger-Gron et al. 2005; Lykke-Andersen and Wagner
2005), suggesting that these processes do not require the presence of visible PBs. Furthermore, in vitro extract that presumably lacks visible structures can recapitulate miRNA-mediated endonucleolytic activity and translational repression (Wang et al. 2006).

Neither PBs nor SGs are confined by membranes, and their components continually exchange with the cytoplasm (Gilks et al. 2004; Yang et al. 2004; Andrei et al. 2005; Kedersha et al. 2005). Given that these proteins are not confined to specific structures and the structures are not required for the translational repression, endonucleolytic activity, or deadenylation/degradation of target mRNA, miRNAs most likely act predominately in the diffuse cytoplasm. This is consistent with the current quantitation data showing that the majority of miRNAs, miRNA targets, and Argonaute proteins are not localized to PBs, but are diffusively distributed in the cytoplasm (Pillai et al. 2005; Leung et al. 2006). Notably, Argonaute family members (dAgo1 and Alg1/2) were also found to be diffusely distributed in the cytoplasm of Drosophila S2 cells and C. elegans epidermal/neuronal cells (Ding et al. 2005; Behm-Ansmant et al. 2006). Only when GW182 homologs were overexpressed did these Argonaute members localize to punctate PB-like structures in the cytoplasm (Ding et al. 2005; Behm-Ansmant et al. 2006). Moreover, GW182 is not always concentrated in mammalian PBs throughout the cell cycle (Yang et al. 2004), and this is also the case for endogenous Argonaute protein (A. Leung, unpubl.). Therefore, the existence of these microscopically visible structures is most likely not required for miRNA function.

\section{So What Are These Structures For?}

The appearance of a specific structure, such as PB, where multiple proteins colocalize, most likely represents complex formation from interactions between different components for a specific pathway or function (Misteli 2001), even though equivalent interactions can probably occur elsewhere in the cytoplasm. As discussed earlier, components in PBs or SGs are continually exchanging with the cytoplasm. In turn, these differential dynamic exchange rates of the protein components should determine their concentration at such structures. Increased local concentration of factors that participate in related steps of a particular process at a physical structure can increase the overall functional efficiency, but the structure itself may not be a prerequisite for the function. Furthermore, visible structures like PBs most likely represent sites for multiple pathways where shared components localize. However, these colocalizations do not necessarily imply functional relationship/dependency. For example, components of the NMD pathway (UPF1), RNAi (dAgo2), and miRNA function (dAgo1) are colocalized in a PB-like structure in Drosophila S2 cells, and knockdown of individual components of one pathway does not affect the function of others (Rehwinkel et al. 2005). These results suggest that a visible structure could be composed of heterogeneous mRNPs. Therefore, structure itself does not imply exclusive functional location, nor does colocalization imply the same functional pathway. 
PB association with miRNA components most likely reflects its role linked to degradation/deadenylation. Previous immunoprecipitation data showed that the associations between Argonaute proteins and other PB components, such as Dcpla (Liu et al. 2005a), rck/p54 (Chu and Rana 2006), and GW182 (Liu et al. 2005b), were due to protein-protein interactions, rather than through a common RNA scaffold. Consistent with this, we observed that the PB association with Argonaute protein does not require miRNAs. Since PBs do not contain either ribosomal subunits or initiation factors, mRNAs interacting with miRNAs at PBs cannot be undergoing repression either at postinitiation or at arrest of initiation processes postbinding of the 40S subunit. Therefore, the ribosome-free PBs are most likely not the sites where active miRNA-mediated translational repression occurs. However, it is possible that the observed stable association of Argonaute protein and PBs, through protein-protein interactions, reinforces a repressive state by keeping the bound message in a translation-incompetent environment. This may be the case for other structures that could simply sequestrate miRNAs from active function in polarized cell types. For example, neurons, which have long cytoplasmic extensions, are known to require specialized machineries to transport mRNAs to dendrites. It remains unclear whether localization of miRNA at dendrites, such as those reported by Schratt et al. (2006), requires specialized structures or is a consequence of miRNA binding to mRNA that is targeted to dendrites.

In light of these results, miRNA-mediated translational repression is likely associated with other complexes in the diffuse cytoplasm. By limiting translation initiation, we identify a pool of Argonaute protein in the cytoplasm that continually exchanges with ribosomes. In this new steady state, the ribosome-associated Argonaute pool dynamically relocates to newly assembled SGs, which contain translationally stalled mRNAs, in a miRNA-dependent manner. We propose here that the pool localized to SGs most likely represents a subset of Argonaute proteins formerly involved in miRNA-mediated translational repression.

\section{FINAL WORDS}

In summary, the majority of Argonaute protein is diffusively distributed in the cytoplasm. Argonaute proteins localized at PBs and SGs are not static but are continually exchanging with the cytoplasm at different rates. Translational repression, endonucleolytic cleavage, and deadenylation remain functional in the absence of microscopically visible PBs and SGs. These results suggest that miRNA-mediated processes can occur in submicroscopic complexes, but not necessarily in distinct subcellular structures, in the cytoplasm.

\section{ACKNOWLEDGMENTS}

We thank Mary Wernett for preparing the illustrations and Alla Grishok, Joel Neilson, Amy White, and Lourdes Aleman in the Sharp Lab for comments on the manuscript, and Tun-Han Leung for proofreading. A.K.L.L. is a recipient of a Human Frontier Science Program long-term fel- lowship. This work was supported by Program Project grant PO1 CA42063 from the National Cancer Institute, by National Science Foundation grant 0218506 to P.A.S., and partially by Cancer Center Support (core) grant P30CA14051 from the National Cancer Institute.

\section{REFERENCES}

Anderson P. and Kedersha N. 2006. RNA granules. J. Cell Biol. 172: 803 .

Andrei M.A., Ingelfinger D., Heintzmann R., Achsel T., RiveraPomar R., and Luhrmann R. 2005. A role for eIF4E and eIF4E-transporter in targeting mRNPs to mammalian processing bodies. RNA 11: 717 .

Arasu P., Wightman B., and Ruvkun G. 1991. Temporal regulation of lin-14 by the antagonistic action of two other heterochronic genes, lin-4 and lin-28. Genes Dev. 5: 1825.

Bagga S., Bracht J., Hunter S., Massirer K., Holtz J., Eachus R., and Pasquinelli A.E. 2005. Regulation by let-7 and lin-4 miRNAs results in target mRNA degradation. Cell 122: 553.

Bartel D.P. 2004. MicroRNAs: Genomics, biogenesis, mechanism, and function. Cell 116: 281.

Behm-Ansmant I., Rehwinkel J., Doerks T., Stark A., Bork P., and Izaurralde E. 2006. mRNA degradation by miRNAs and GW182 requires both CCR4:NOT deadenylase and DCP1:DCP2 decapping complexes. Genes Dev. 20: 1885.

Bhattacharyya S.N., Habermacher R., Martine U., Closs E.I., and Filipowicz W. 2006. Relief of microRNA-mediated translational repression in human cells subjected to stress. Cell 125: 1111.

Birmingham A., Anderson E.M., Reynolds A., Ilsley-Tyree D., Leake D., Fedorov Y., Baskerville S., Maksimova E., Robinson K., Karpilow J., et al. 2006. 3' UTR seed matches, but not overall identity, are associated with RNAi off-targets. Nat. Methods 3: 199.

Brengues M., Teixeira D., and Parker R. 2005. Movement of eukaryotic mRNAs between polysomes and cytoplasmic processing bodies. Science 310: 486.

Brennecke J., Hipfner D.R., Stark A., Russell R.B., and Cohen S.M. 2003. bantam encodes a developmentally regulated microRNA that controls cell proliferation and regulates the proapoptotic gene hid in Drosophila. Cell 113: 25.

Carmell M.A., Xuan Z., Zhang M.Q., and Hannon G.J. 2002. The Argonaute family: Tentacles that reach into RNAi, developmental control, stem cell maintenance, and tumorigenesis. Genes Dev. 16: 2733.

Caudy A.A., Myers M., Hannon G.J., and Hammond S.M. 2002. Fragile X-related protein and VIG associate with the RNA interference machinery. Genes Dev. 16: 2491.

Chen C.Z., Li L., Lodish H.F., and Bartel D.P. 2004. MicroRNAs modulate hematopoietic lineage differentiation. Science 303: 83.

Chu C.Y. and Rana T.M. 2006. Translation repression in human cells by microRNA-induced gene silencing requires RCK/p54. PLoS Biol. 4: e210.

Cimmino A., Calin G.A., Fabbri M., Iorio M.V., Ferracin M., Shimizu M., Wojcik S.E., Aqeilan R.I., Zupo S., Dono M., et al. 2005. miR-15 and miR-16 induce apoptosis by targeting BCL2. Proc. Natl. Acad. Sci. 102: 13944.

Clark I.E., Wyckoff D., and Gavis E.R. 2000. Synthesis of the posterior determinant Nanos is spatially restricted by a novel cotranslational regulatory mechanism. Curr. Biol. 10: 1311.

Cougot N., Babajko S., and Seraphin B. 2004. Cytoplasmic foci are sites of mRNA decay in human cells. J. Cell Biol. 165: 31 .

Davis E., Caiment F., Tordoir X., Cavaille J., Ferguson-Smith A., Cockett N., Georges M., and Charlier C. 2005. RNAimediated allelic trans-interaction at the imprinted Rtl1/Peg11 locus. Curr. Biol. 15: 743.

Ding L., Spencer A., Morita K., and Han M. 2005. The developmental timing regulator AIN-1 interacts with miRISCs and may target the argonaute protein ALG-1 to cytoplasmic P bodies in C. elegans. Mol. Cell 19: 437. 
Doench J.G. and Sharp P.A. 2004. Specificity of microRNA target selection in translational repression. Genes Dev. 18: 504.

Doench J.G., Petersen C.P., and Sharp P.A. 2003. siRNAs can function as miRNAs. Genes Dev. 17: 438.

Farh K.K., Grimson A., Jan C., Lewis B.P., Johnston W.K., Lim L.P., Burge C.B., and Bartel D.P. 2005. The widespread impact of mammalian microRNAs on mRNA repression and evolution. Science 310: 1817

Fedorov Y., Anderson E.M., Birmingham A., Reynolds A., Karpilow J., Robinson K., Leake D., Marshall W.S., and Khvorova A. 2006. Off-target effects by siRNA can induce toxic phenotype. RNA 12: 1188.

Fenger-Gron M., Fillman C., Norrild B., and Lykke-Andersen J. 2005. Multiple processing body factors and the ARE binding protein TTP activate mRNA decapping. Mol. Cell 20: 905

Filipowicz W., Jaskiewicz L., Kolb F.A., and Pillai R.S. 2005. Post-transcriptional gene silencing by siRNAs and miRNAs. Curr. Opin. Struct. Biol. 15: 331.

Fillman C. and Lykke-Andersen J. 2005. RNA decapping inside and outside of processing bodies. Curr. Opin. Cell Biol. 17: 326.

Gavis E.R. and Lehmann R. 1994. Translational regulation of nanos by RNA localization. Nature 369: 315 .

Gebauer F. and Hentze M.W. 2004. Molecular mechanisms of translational control. Nat. Rev. Mol. Cell Biol. 5: 827.

Gilks N., Kedersha N., Ayodele M., Shen L., Stoecklin G., Dember L.M., and Anderson P. 2004. Stress granule assembly is mediated by prion-like aggregation of TIA-1. Mol. Biol. Cell 15: 5383.

Giraldez A.J., Mishima Y., Rihel J., Grocock R.J., Van Dongen S., Inoue K., Enright A.J., and Schier A.F. 2006. Zebrafish MiR-430 promotes deadenylation and clearance of maternal mRNAs. Science 312: 75.

Gupta A., Gartner J.J., Sethupathy P., Hatzigeorgiou A.G., and Fraser N.W. 2006. Anti-apoptotic function of a microRNA encoded by the HSV-1 latency-associated transcript. Nature 442: 82 .

Ha I., Wightman B., and Ruvkun G. 1996. A bulged lin-4/lin-14 RNA duplex is sufficient for Caenorhabditis elegans lin-14 temporal gradient formation. Genes Dev. 10: 3041.

Hornstein E., Mansfield J.H., Yekta S., Hu J.K., Harfe B.D., McManus M.T., Baskerville S., Bartel D.P., and Tabin C.J. 2005. The microRNA miR-196 acts upstream of Hoxb8 and Shh in limb development. Nature 438: 671.

Humphreys D.T., Westman B.J., Martin D.I., and Preiss T. 2005. MicroRNAs control translation initiation by inhibiting eukaryotic initiation factor 4E/cap and poly(A) tail function. Proc. Natl. Acad. Sci. 102: 16961

Hutvagner G. and Zamore P.D. 2002. A microRNA in a multiple-turnover RNAi enzyme complex. Science 297: 2056.

Jackson A.L., Burchard J., Schelter J., Chau B.N., Cleary M., Lim L., and Linsley P.S. 2006a. Widespread siRNA "off-target" transcript silencing mediated by seed region sequence complementarity. RNA 12: 1179.

Jackson A.L., Bartz S.R., Schelter J., Kobayashi S.V., Burchard J., Mao M., Li B., Cavet G., and Linsley P.S. 2003. Expression profiling reveals off-target gene regulation by RNAi. Nat. Biotechnol. 21: 635

Jackson A.L., Burchard J., Leake D., Reynolds A., Schelter J., Guo J., Johnson J.M., Lim L., Karpilow J., Nichols K., et al. 2006b. Position-specific chemical modification of siRNAs reduces "off-target" transcript silencing. RNA 12: 1197.

Jakymiw A., Lian S., Eystathioy T., Li S., Satoh M., Hamel J.C., Fritzler M.J., and Chan E.K. 2005. Disruption of GW bodies impairs mammalian RNA interference. Nat. Cell Biol. 7: 1267.

Jing Q., Huang S., Guth S., Zarubin T., Motoyama A., Chen J., Di Padova F., Lin S.C., Gram H., and Han J. 2005. Involvement of microRNA in AU-rich element-mediated mRNA instability. Cell 120: 623.

Kedersha N. and Anderson P. 2002. Stress granules: Sites of mRNA triage that regulate mRNA stability and translatability. Biochem. Soc. Trans. 30: 963
Kedersha N., Stoecklin G., Ayodele M., Yacono P., LykkeAndersen J., Fitzler M.J., Scheuner D., Kaufman R.J., Golan D.E., and Anderson P. 2005. Stress granules and processing bodies are dynamically linked sites of mRNP remodeling. J. Cell Biol. 169: 871 .

Kim J., Krichevsky A., Grad Y., Hayes G.D., Kosik K.S., Church G.M., and Ruvkun G. 2004. Identification of many microRNAs that copurify with polyribosomes in mammalian neurons. Proc. Natl. Acad. Sci. 101: 360.

Kim V.N. 2005. MicroRNA biogenesis: Coordinated cropping and dicing. Nat. Rev. Mol. Cell Biol. 6: 376.

Lee R.C., Feinbaum R.L., and Ambros V. 1993. The C. elegans heterochronic gene lin-4 encodes small RNAs with antisense complementarity to lin-14. Cell 75: 843.

Leung A.K.L., Calabrese J.M., and Sharp P.A. 2006. Quantitative analysis of Argonaute protein reveals microRNA-dependent localization to stress granules. Proc. Natl. Acad. Sci. 103: 18125.

Lewis B.P., Burge C.B., and Bartel D.P. 2005. Conserved seed pairing, often flanked by adenosines, indicates that thousands of human genes are microRNA targets. Cell 120: 15 .

Lim L.P., Lau N.C., Garrett-Engele P., Grimson A., Schelter J.M., Castle J., Bartel D.P., Linsley P.S., and Johnson J.M. 2005. Microarray analysis shows that some microRNAs downregulate large numbers of target mRNAs. Nature 433: 769.

Lin X., Ruan X., Anderson M.G., McDowell J.A., Kroeger P.E., Fesik S.W., and Shen Y. 2005. siRNA-mediated offtarget gene silencing triggered by a $7 \mathrm{nt}$ complementation. Nucleic Acids Res. 33: 4527.

Lippincott-Schwartz J., Altan-Bonnet N., and Patterson G.H. 2003. Photobleaching and photoactivation: Following protein dynamics in living cells. Nat. Cell Biol. (suppl.) S7.

Liu J., Valencia-Sanchez M.A., Hannon G.J., and Parker R. 2005a. MicroRNA-dependent localization of targeted mRNAs to mammalian P-bodies. Nat. Cell Biol. 7: 719.

Liu J., Rivas F.V., Wohlschlegel J., Yates J.R., III, Parker R., and Hannon G.J. 2005b. A role for the P-body component GW182 in microRNA function. Nat. Cell Biol. 7: 1261.

Liu J., Carmell M.A., Rivas F.V., Marsden C.G., Thomson J.M., Song J.J., Hammond S.M., Joshua-Tor L., and Hannon G.J. 2004. Argonaute2 is the catalytic engine of mammalian RNAi. Science 305: 1437.

Lykke-Andersen J. and Wagner E. 2005. Recruitment and activation of mRNA decay enzymes by two ARE-mediated decay activation domains in the proteins TTP and BRF-1. Genes Dev. 19: 351.

Misteli T. 2001. The concept of self-organization in cellular architecture. J. Cell Biol. 155: 181.

Nelson P.T., Hatzigeorgiou A.G., and Mourelatos Z. 2004. miRNP:mRNA association in polyribosomes in a human neuronal cell line. RNA 10: 387 .

Olsen P.H. and Ambros V. 1999. The lin-4 regulatory RNA controls developmental timing in Caenorhabditis elegans by blocking LIN-14 protein synthesis after the initiation of translation. Dev. Biol. 216: 671.

Parker R. and Song H. 2004. The enzymes and control of eukaryotic mRNA turnover. Nat. Struct. Mol. Biol. 11: 121.

Pestova T.V., Kolupaeva V.G., Lomakin I.B., Pilipenko E.V., Shatsky I.N., Agol V.I., and Hellen C.U. 2001. Molecular mechanisms of translation initiation in eukaryotes. Proc. Natl. Acad. Sci. 98: 7029.

Petersen C.P., Bordeleau M.E., Pelletier J., and Sharp P.A. 2006. Short RNAs repress translation after initiation in mammalian cells. Mol. Cell 21: 533.

Pillai R.S., Bhattacharyya S.N., Artus C.G., Zoller T., Cougot N., Basyuk E., Bertrand E., and Filipowicz W. 2005. Inhibition of translational initiation by Let-7 microRNA in human cells. Science 309: 1573.

Poy M.N., Eliasson L., Krutzfeldt J., Kuwajima S., Ma X., Macdonald P.E., Pfeffer S., Tuschl T., Rajewsky N., Rorsman P., and Stoffel M. 2004. A pancreatic islet-specific microRNA regulates insulin secretion. Nature 432: 226 . 
Rajewsky N. 2006. microRNA target predictions in animals. Nat. Genet. (suppl. 1) 38: S8.

Rehwinkel J., Behm-Ansmant I., Gatfield D., and Izaurralde E. 2005. A crucial role for GW182 and the DCP1:DCP2 decapping complex in miRNA-mediated gene silencing. RNA 11: 1640 .

Rehwinkel J., Natalin P., Stark A., Brennecke J., Cohen S.M., and Izaurralde E. 2006. Genome-wide analysis of mRNAs regulated by Drosha and Argonaute proteins in Drosophila melanogaster. Mol. Cell. Biol. 26: 2965.

Rivas F.V., Tolia N.H., Song J.J., Aragon J.P., Liu J., Hannon G.J., and Joshua-Tor L. 2005. Purified Argonaute2 and an siRNA form recombinant human RISC. Nat. Struct. Mol. Biol. 12: 340 .

Saxena S., Jonsson Z.O., and Dutta A. 2003. Small RNAs with imperfect match to endogenous mRNA repress translation. Implications for off-target activity of small inhibitory RNA in mammalian cells. J. Biol. Chem. 278: 44312.

Schratt G.M., Tuebing F., Nigh E.A., Kane C.G., Sabatini M.E., Kiebler M., and Greenberg M.E. 2006. A brain-specific microRNA regulates dendritic spine development. Nature 439: 283 .

Sen G.L. and Blau H.M. 2005. Argonaute 2/RISC resides in sites of mammalian mRNA decay known as cytoplasmic bodies. Nat. Cell Biol. 7: 633.

Sheth U. and Parker R. 2003. Decapping and decay of messenger RNA occur in cytoplasmic processing bodies. Science 300: 805 .

Sonenberg N. and Dever T.E. 2003. Eukaryotic translation initiation factors and regulators. Curr. Opin. Struct. Biol. 13: 56.
Teixeira D., Sheth U., Valencia-Sanchez M.A., Brengues M., and Parker R. 2005. Processing bodies require RNA for assembly and contain nontranslating mRNAs. RNA 11: 371 .

Tomari Y., Matranga C., Haley B., Martinez N., and Zamore P.D. 2004. A protein sensor for siRNA asymmetry. Science 306: 1377.

Valencia-Sanchez M.A., Liu J., Hannon G.J., and Parker R. 2006. Control of translation and mRNA degradation by miRNAs and siRNAs. Genes Dev. 20: 515.

Wang B., Love T.M., Call M.E., Doench J.G., and Novina C.D. 2006. Recapitulation of short RNA-directed translational gene silencing in vitro. Mol. Cell 22: 553.

Wightman B., Ha I., and Ruvkun G. 1993. Posttranscriptional regulation of the heterochronic gene lin-14 by lin- 4 mediates temporal pattern formation in C. elegans. Cell 75: 855.

$\mathrm{Wu}$ L. and Belasco J.G. 2005. Micro-RNA regulation of the mammalian lin-28 gene during neuronal differentiation of embryonal carcinoma cells. Mol. Cell. Biol. 25: 9198.

Wu L., Fan J., and Belasco J.G. 2006. MicroRNAs direct rapid deadenylation of mRNA. Proc. Natl. Acad. Sci. 103: 4034.

Yang Z., Jakymiw A., Wood M.R., Eystathioy T., Rubin R.L., Fritzler M.J., and Chan E.K. 2004. GW182 is critical for the stability of GW bodies expressed during the cell cycle and cell proliferation. J. Cell Sci. 117: 5567.

Yekta S., Shih I.H., and Bartel D.P. 2004. MicroRNA-directed cleavage of HOXB8 mRNA. Science 304: 594.

Zeng Y., Yi R., and Cullen B.R. 2003. MicroRNAs and small interfering RNAs can inhibit mRNA expression by similar mechanisms. Proc. Natl. Acad. Sci. 100: 9779. 


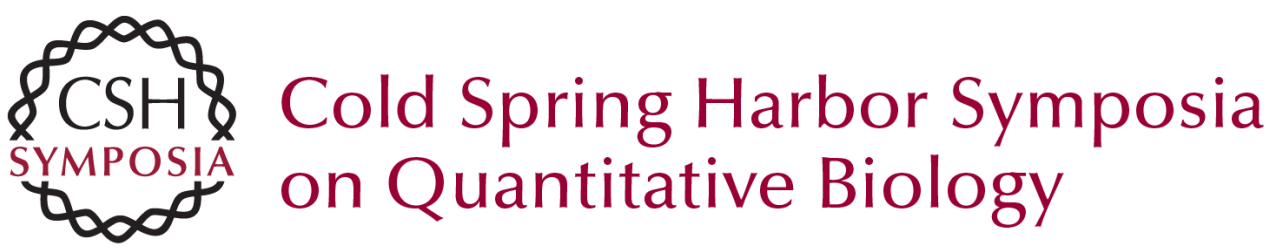

\section{Function and Localization of MicroRNAs in Mammalian Cells}

A.K.L. LEUNG and P.A. SHARP

Cold Spring Harb Symp Quant Biol 2006 71: 29-38

Access the most recent version at doi:10.1101/sqb.2006.71.049

References This article cites 80 articles, 42 of which can be accessed free at: http://symposium.cshlp.org/content/71/29.full.html\#ref-list-1

License

Email Alerting Receive free email alerts when new articles cite this article - sign up in Service the box at the top right corner of the article or click here. 